\title{
Fine-Grained Tracker as a Near Detector for LBNE
}

\author{
Xinchun Tian*, Sanjib Mishra, Roberto Petti and Duyang Hongyue \\ University of South Carolina \\ E-mail: tianxc@gmail.com
}

\begin{abstract}
The reference design of the near detector for the LBNE experiment is a high-resolution FineGrained Tracker (FGT) capable of precisely measuring all four species of neutrinos: $v_{\mu}, v_{e}, \bar{v}_{\mu}$ and $\bar{v}_{e}$. The FGT is composed of a Straw-Tube Tracker (STT) with transition-radiation capability surrounded by a high resolution electromagnetic calorimeter (ECAL) and embedded in a dipole magnet. Muon-ID detectors instrument the iron-yoke of the magnet and the downstream and upstream stations outside the magnet. The STT is instrumented with Ar and other nuclear targets. The goals of the FGT is to constrain the systematic errors, below the corresponding statistical error in the far detector, for all oscillation studies; and to conduct a panoply of precision measurements and searches in Neutrino physics. We present sensitivity studies - critical to constraining the systematics in oscillation searches - of measurements of (1) the absolute neutrino flux, (2) neutrino-nucleon quasi-elastic (QE) and (3) resonance (Res) interactions. In QE and Res emphasis is laid in identifying in situ measurables that help constrain nuclear effects such as initial state pair wise correlations and final state interactions.
\end{abstract}

16th International Workshop on Neutrino Factories and Future Neutrino Beam Facilities 25 -30 August, 2014

University of Glasgow, United Kingdom

${ }^{*}$ Speaker. 


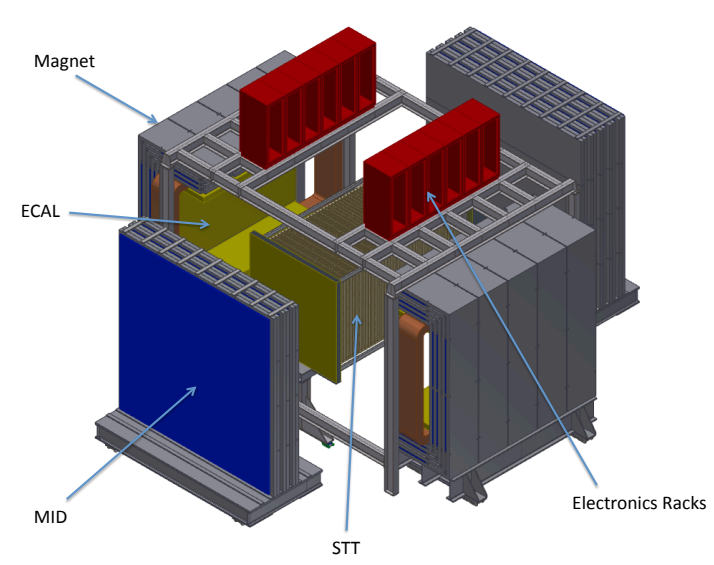

Figure 1: The LBNE near neutrino detector reference design - Fine-Grained Tracker.

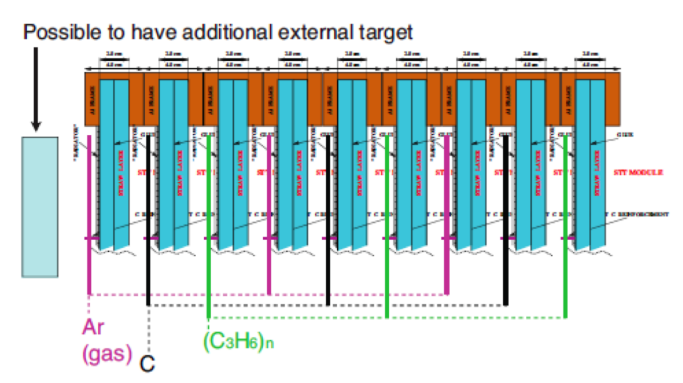

Figure 2: High-pressure argon gas targets, as well as water and other nuclear targets, are embedded in the upstream part of the tracking volume.

\section{LBNE/F Near Detector}

The reference design of the near detector for the LBNE experiment shown in Fig. 1 is a highresolution Fine-Grained Tracker (FGT) capable of precisely measuring all four species of neutrinos: $v_{\mu}, v_{e}, \bar{v}_{\mu}$ and $\bar{v}_{e}$. The FGT is composed of a $3 \times 3 \times 7.04 \mathrm{~m}^{3}$ Straw-Tube Tracker (STT) with transition-radiation capability surrounded by a high resolution electromagnetic calorimeter (ECAL) and embedded in a 0.4-T dipole magnet. Muon-ID detectors instrument the iron-yoke of the magnet and the downstream and upstream stations outside the magnet. The STT is instrumented with Ar and other nuclear targets as shown in Fig. 2 leading to an accurate modeling of neutrino-nucleus interactions. Table 1 summarizes the performance for the fine-grained tracker's configuration, and Table 2 lists its parameters. The goals of the FGT is to constrain the systematic errors, below the corresponding statistical error in the far detector, for all oscillation studies; and to conduct a panoply of precision measurements and searches in Neutrino physics.

In the following sections, We will present sensitivity studies - critical to constraining the systematics in oscillation searches - of measurements of (1) the absolute neutrino flux, (2) neutrinonucleon quasi-elastic (QE) and (3) resonance (Res) interactions using a parametrized fast Monte Carlo simulation. In QE and Res emphasis is laid in identifying in situ measurables that help constrain nuclear effects such as initial state pair wise correlations and final state interactions.

\section{Fast Monte Carlo}

A Fast Monte Carlo simulation has been developed which combines the Geant4 LBNE beamline flux predictions, the GENIE [2] event interaction generator, and a parameterized detector response that is used to simulate the measured (reconstructed) energy and momentum of each final-state particle. The magnetic field is simulated using the classical $4^{\text {th }}$ order Runge-Kutta stepper which is the default method in Geant 4 to compute the motion of a charged track in a general field. For charged particles, the momentum resolution is determined by $\sigma_{p} / p=\frac{0.05}{\sqrt{L}}+$ $0.008 p / \sqrt{L^{5}}$, where $p$ is momentum and $L$ is track length; the angular resolution is determined 


\begin{tabular}{|c|c|}
\hline $\begin{array}{l}\text { Vertex Resolution } \\
\text { Angular Resolution }\end{array}$ & $\begin{array}{l}0.1 \mathrm{~mm} \\
2 \mathrm{mrad}\end{array}$ \\
\hline $\begin{array}{l}E_{e} \text { Resolution } \\
E_{\mu} \text { Resolution }\end{array}$ & $\begin{array}{c}6 \% / \sqrt{E}(\sim 4 \% \text { at } 3 \mathrm{GeV}) \\
3.5 \%\end{array}$ \\
\hline$v_{\mu} / \bar{v}_{\mu}$ ID & Yes \\
\hline$v_{e} / \bar{v}_{e} \mathrm{ID}$ & Yes \\
\hline$\pi^{-}$.vs. $\pi^{+}$ID & Yes \\
\hline$\pi^{+}$.vs. proton .vs. $K^{+}$ & Yes \\
\hline $\mathrm{NC} \pi^{0} / \mathrm{CC} e$ Rejection & $0.1 \%$ \\
\hline $\mathrm{NC} \gamma / \mathrm{CC} e$ Rejection & $0.2 \%$ \\
\hline $\mathrm{CC} \mu / \mathrm{CC} e$ Rejection & $0.01 \%$ \\
\hline
\end{tabular}

Table 1: Summary of the performance for the Fine-Grained Tracker configuration.

\begin{tabular}{l|l}
\hline STT detector volume & $3 \times 3 \times 7.04 \mathrm{~m}^{3}$ \\
STT detector mass & 8 tons \\
Number of straws in STT & 123,904 \\
Inner magnetic volume & $4.5 \times 4.5 \times 8.0 \mathrm{~m}^{3}$ \\
Targets & $1.27 \mathrm{~cm}$ thick argon $(\sim 50 \mathrm{~kg})$, water and others \\
Transition radiation radiators & $2.5 \mathrm{~cm}$ thick \\
ECAL $X_{0}$ & 10 barrel, 10 backward, 18 forward \\
Number of scintillator bars in ECAL & 32,320 \\
Dipole magnet & $2.4 \mathrm{MW}$ power; $60 \mathrm{~cm}$ steel thickness \\
Magnetic field and uniformity & $0.4 \mathrm{~T} ;<2 \%$ variation over inner volume \\
MuID configuration & $32 \mathrm{RPC}$ planes interspersed between $20 \mathrm{~cm}$ thick layers of steel \\
\hline
\end{tabular}

Table 2: Parameters for the Fine-Grained Tracker.

by $\theta_{0}=\frac{13.6 \mathrm{MeV}}{\beta c p} z \sqrt{x / X_{0}}\left[1+0.038 \ln \left(x / X_{0}\right)\right]$ where $p, \beta c$, and $z$ are the momentum, velocity, and charge number of the incident particle, and $x / X_{0}$ is the thickness of the scattering medium in radiation lengths [3]. The radiation length is $X_{0}=600 \mathrm{~cm}$, and the electromagnetic and hadronic shower energy resolution are parametrized by $\sigma_{E} / E=1 \%+0.06 / \sqrt{E}$ and $\sigma_{E} / E=1 \%+0.50 / \sqrt{E}$, respectively.

\section{Absolute Neutrino Flux}

The high-resolution Fine-Grained Tracker can accurately measuring the un-oscillated beam flux with a precision $\leq 2 \%$ for absolute normalization with neutrino-electron $\mathrm{NC}(\mathrm{CC})$ scattering.

\subsection{Low-Energy Absolute Flux: Neutrino-Electron NC Scattering}

Neutrino neutral current (NC) interaction with the atomic electron in the target, $v e^{-} \rightarrow v e^{-}$, provides an elegant measure of the absolute flux. The total cross section for NC elastic scattering 
off atom electrons is given in Ref. [4]: The cross section of neutrino-electron neutral current is very small, $\sim 10^{-42}\left(E_{V}\right) / \mathrm{GeV} \mathrm{cm}{ }^{2}$, which is $\sim 2000$ times smaller than that for neutrino-nucleon scattering. The cross section only depends on the precision of $\sin ^{2} \theta_{W}$, for LBNE, the precision is $\leq 1 \%$. Assuming $1.2 \mathrm{MW}$ beam power, 5 tons of fiducial volume and 3 years running, the expected signal events are approximately 6,300 .

The signature of neutrino-electron $\mathrm{NC}$ scattering is a single $e^{-}$collinear with the neutrino beam ( $\leq 40 \mathrm{mrad})$. The following selection criteria are applied to select the signal and reject the background events: 1) fiducial volume, 2) one negative charged track, no positive track(s), 3) electron momentum $p_{e}>0.5 \mathrm{GeV}$, 4) electron track with minimum number of hits $n_{e}^{\text {hits }} \geq 12$, 5) electron transverse momentum $\left.p_{e}^{T}<0.1 \mathrm{GeV}, 6\right)$ electron angle with respect to the neutrino beam $\theta_{e}<0.1 \mathrm{rad}$. The events with identified muon and/or $\pi^{0} / n / K_{0}$ will be further rejected. At the end of the selection, the signal efficiency is $73 \%$ with benign background which comes from $v_{e}$ CCQE and asymmetric conversion of a photon in an ordinary neutrino-nucleon NC events producing $e^{-}$and $e^{+}$in equal measure with much broader angular distribution. The background can be constrained in-situ by selecting the $e^{+}$events. There are $\sim 3200$ neutrino-electron NC signal events passing all the selection criteria. The determination of the absolute flux is estimated to reach a precision of $\simeq 2.5 \%$ for $E_{v}<10 \mathrm{GeV}$, and the measurement will be dominated by the statistical error. The measurement of NC elastic scattering off electrons can only provide the integral of all neutrino flavors. Recently, MINER $v$ A collaboration reported a precision of $13 \%$ in absolute flux determination has been achieved [5].

\subsection{High-Energy Absolute Flux: Neutrino-Electron CC Scattering (IMD)}

The neutrino-electron CC interaction, $v_{l}+e^{-} \rightarrow l^{-}+v_{e}$ (inverse muon decay or IMD), offers an elegant way to determine the absolute neutrino flux. The total cross section for CC elastic scattering off atom electrons is given in Ref. [4]: The threshold (for electrons at rest) $E_{v} \geq \frac{m_{l}^{2}-m_{e}^{2}}{2 m_{e}}$ is very high, for $l=\mu, E_{v} \geq 10.8 \mathrm{GeV}$. Assuming 1.2 MW beam power, 5 tons of fiducial volume and 3 years running, the expected signal events are around 3,200 .

Similiar to the neutrino-electron NC scattering, the signature of IMD is a single $\mu^{-}$collinear with the neutrino beam. The following selection criteria are applied to select the signal and reject the background: 1) fiducial volume, 2) one negative charged track identified as a muon, no positive $\operatorname{track}(\mathrm{s}), 3)$ muon energy $\left.E_{\mu}>10.9 \mathrm{GeV}, 4\right)$ muon track with minimum number of hits $n_{\mu}^{\text {hits }} \geq 12$, 5) muon transverse momentum $\left.p_{\mu}^{T}<0.15 \mathrm{GeV}, 6\right)$ muon angle with respect to the neutrino beam $\left.\theta_{\mu}<0.005 \mathrm{rad}, 7\right) \mathscr{Z}_{\mu}=E_{\mu}\left(1-\cos \theta_{\mu}\right)<0.00025 \mathrm{GeV}$. The events with identified $\pi^{0} / n / K_{0}$ will be further rejected. At the end of the selection, the signal efficiency is $93 \%$ with $26 \%$ background which is dominated by 1 track $v_{\mu}$ CCQE. The background can be constrained in-situ using CCQE 2 track control samples, when the hadronic track is removed. The systematic limitations of CCFR [6, 7] and the CHARM-II [8] IMD measurements using the $\mu^{+}$sample can be substantially alleviated in LBNE with the proposed Fine-Grained Tracker design. There are $\sim 3000$ neutrino-electron CC signal events passing the selection criteria, and the determination of the absolute flux is estimated to reach a precision of $\simeq 3.0 \%$ for $E_{v}>11 \mathrm{GeV}$.

Neutrino-electron NC (CC) scattering is a simple two-body interaction, therefore using the electron (muon) momentum and angle, we can derive the incoming neutrino energy. Figs. 3 show the comparison between the true neutrino energy (blue curve) with the derived neutrino energy 

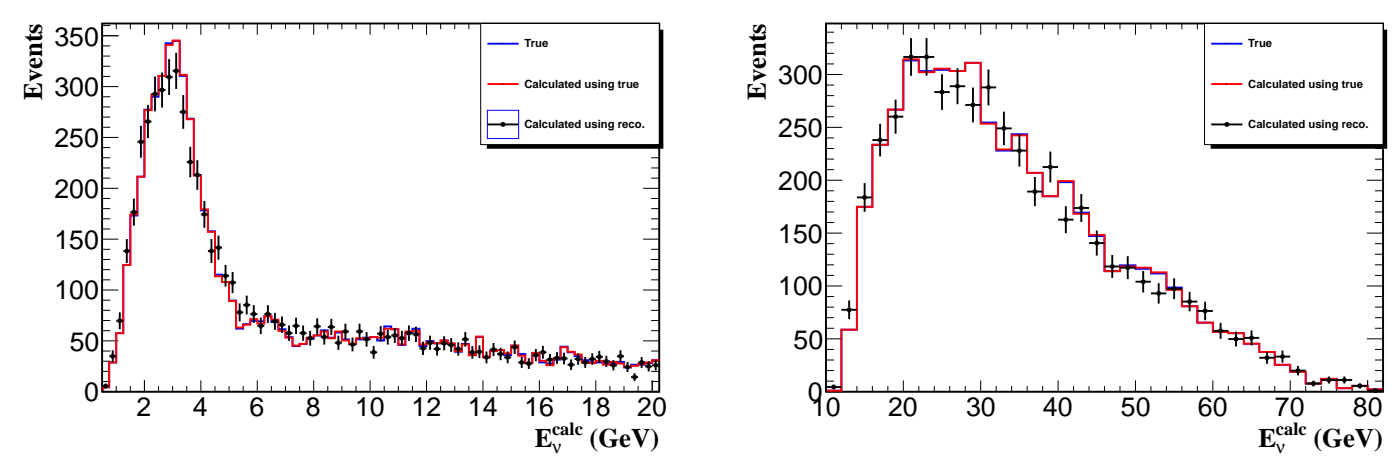

Figure 3: The derived neutrino energy using the emergent electron (left) and muon (right) momentum and angle.

(black dots) for neutrino-electron NC (left) and CC (right) scattering. The plots show that the neutrino-electron $\mathrm{NC}$ (CC) scattering can not only determine the neutrino absolute flux normalization to high precision, but also the flux shape.

\section{Cross Sections and Nuclear Models: Charged-Current Quasi-Elastic}

The Charged-Current Quasi-Elastic (CCQE) process plays an important role in the LBNE oscillation physics. Precise measurements of QE will impose direct constraints on the systematic uncertainties in neutrino-nucleon cross sections and nuclear models.

In CCQE, events will be classified as two-track sample when both muon and proton track are successfully reconstructed and one-track sample when only the muon is reconstructed. For the two-track sample, the following selection criteria are applied to select the signal and reject the background events: 1) fiducial volume 2) one negative charged track identified as a muon, 3) muon momentum $p_{\mu}>0.2 \mathrm{GeV}$, 4) muon track with minimum number of hits $n_{e}^{\text {hits }} \geq 12$, 5) proton momentum $\left.p_{\text {proton }}>0.1 \mathrm{GeV}, 6\right)$ proton track with minimum number of hits $n_{\text {proton }}^{\text {hits }} \geq 4$. The events with identified $\pi^{0} / n / K_{0}$ will be further rejected. At the end of the selection, the signal efficiency is $56 \%$ and the purity is $48 \%$. The remaining event sample, composed of muon and proton, is subjected to a multivariate analysis using artificial neural network that maximizes the signal (CCQE) efficiency while keeping the Res/DIS backgrounds low by exploiting the kinematic relation between the muon and proton vectors. The energy-averaged efficiency (purity) is $48 \%$ (76\%) when placing a cut at 0.45 on the multivariate distribution. Fig. 4 (left) shows the expected precision of CCQE cross section obtained in two-track analysis in brown triangle with MiniBooNE [9], NOMAD measurements [10] and the prediction from GENIE. As a a two-body interaction, the incident neutrino energy $\left(E_{v}^{\text {calc }}\right)$ can be derived by using the energy and angle of the muon, $\left(E_{\mu}\right.$ and $\left.\theta_{\mu}\right)$, and compare it with the total visible energy of the two tracks, i.e., $E_{\mathrm{vis}}$ is $=E_{\mu}+E_{\text {proton }}$; the difference between the $E_{\text {vis }}$ and $E_{v}^{\text {calc }}$ is, to a first approximation, the Fermi-motion. Fig. 5 (left) shows that from the fast Monte Carlo simulation.

$$
E_{v}^{\mathrm{calc}}=\frac{2\left(M_{n}-E_{B}\right) E_{\mu}-\left(E_{B}^{2}-2 M_{n} E_{B}+m_{\mu}^{2}+\delta M^{2}\right)}{2\left[\left(M_{n}-E_{B}\right)-E_{\mu}+p_{\mu} \cos \theta_{\mu}\right]},
$$


where $E_{\mu}=T_{\mu}+m_{\mu}$ is the total muon energy and $M_{n}, m_{\mu}$ are the neutron and muon masses. Fig. 6 shows the $\Delta E$ distribution using two different nuclear models, relativistic Fermi gas model (RFGM) and spectral function (SF) (see Ref. [11] and references therein). The high-resolution Fine-Grained Tracker has the precision to tell which nuclear model describes the data and reject the wrong hypothesis.

For the one-track sample, the selection criteria are very similar to the two-track sample, but no second track. The remaining event sample, composed of single muon, is subjected to a multivariate analysis using artificial neural network. The energy-averaged efficiency (purity) is $23 \%$ (94\%) when placing a cut at 0.11 on the multivariate distribution. Fig. 4 (right) shows the expected precision of CCQE cross section obtained in one-track analysis in grey triangle with MiniBooNE [9], NOMAD measurements [10] and the prediction from GENIE.

\section{Cross Sections and Nuclear Models: Charged-Current Resonance}

The Charge-Current Resonance (CCRes) production is the most abundant process in the LBNE energy region. In order to constraint the oscillation physics, we have to measure the resonance production precisely. CCRes provides another independent handle to constrain the nuclear effects which include initial state pair-wise correlation and final state interaction.

In this study, the three-track CCRes sample is used for the study,

$$
v_{\mu}+p \rightarrow \mu^{-}+\Delta^{++} \rightarrow \mu^{-}+p+\pi^{+}
$$

where both proton and pion are reconstructed with an identified muon track. The following selection criteria are applied to select the signal and reject the background events: 1) fiducial volume, 2) one negative charged track identified as a muon, 3) muon momentum $p_{\mu}>0.2 \mathrm{GeV}$, 4) muon track with minimum number of hits $n_{e}^{\text {hits }} \geq 12,5$ ) proton (pion) momentum $p_{\text {proton }\left(\pi^{+}\right)}>0.1 \mathrm{GeV}$, 6) proton (pion) track with minimum number of hits $n_{\text {proton }\left(\pi^{+}\right)}^{\text {hits }} \geq 4$. The events with identified $\pi^{0} / n / K_{0}$ will be further rejected. At the end of the selection, the signal efficiency is $36 \%$ and the purity is $68 \%$. The remaining event sample, composed of muon, proton and pion, is subjected to a multivariate analysis using artificial neural network that maximizes the signal (CCQE) efficiency while keeping the DIS backgrounds low. The energy-averaged efficiency (purity) is 33\% (77\%) when placing a cut at 0.35 on the multivariate distribution. Fig. 4 (left) shows the expected precision of CCRes cross section obtained in three-track analysis in blue dots with Gargamelle [12], SKAT measurements $[13,14]$ and the prediction from GENIE.

The similar technique applied in two-track CCQE can also be used in three-track CCRes to constrain the Fermi-motion. The incident neutrino energy $\left(E_{v}^{\text {calc }}\right)$ can be derived by using the energy and angle of the muon, $\left(E_{\mu}\right.$ and $\left.\theta_{\mu}\right)$, and compare it with the total visible energy of the two tracks, i.e., $E_{\mathrm{vis}}$ is $=E_{\mu}+E_{\mathrm{proton}}+E_{\pi}$; the difference between the $E_{\mathrm{vis}}$ and $E_{v}^{\text {calc }}$ is, to a first approximation, the Fermi-motion. Fig. 5 (right) shows that from the fast Monte Carlo simulation.

$$
E_{v}=\frac{m_{\mu}^{2}+m_{\pi}^{2}-2 M_{p}\left(E_{\mu}+E_{\pi}\right)+2 p_{\mu} \cdot p_{\pi}}{2\left(E_{\mu}+E_{\pi}-\left|\mathbf{P}_{\mu}\right| \cos \theta_{\mu}-\left|\mathbf{P}_{\pi}\right| \cos \theta_{\pi}-M_{p}\right)},
$$

where $E_{\mu}=T_{\mu}+m_{\mu}$ is the total muon energy and $M_{p}, m_{\mu}, m_{\pi}$ are the proton, muon and pion masses. By comparing this distribution to the data we can constrain the nuclear modeling in the neutrino event generator [15]. 

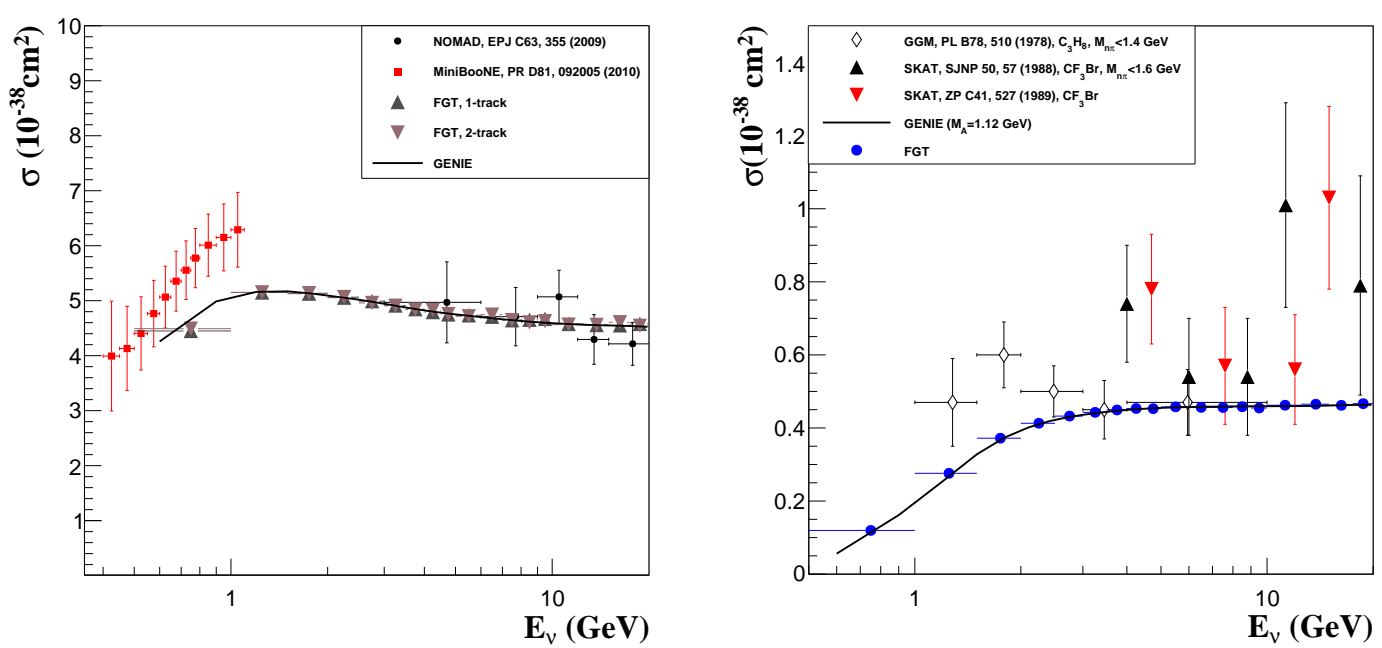

Figure 4: The expected cross section in Charged-Current Quasi-Elastic (left) and Charged-Current 3 track resonance (right).
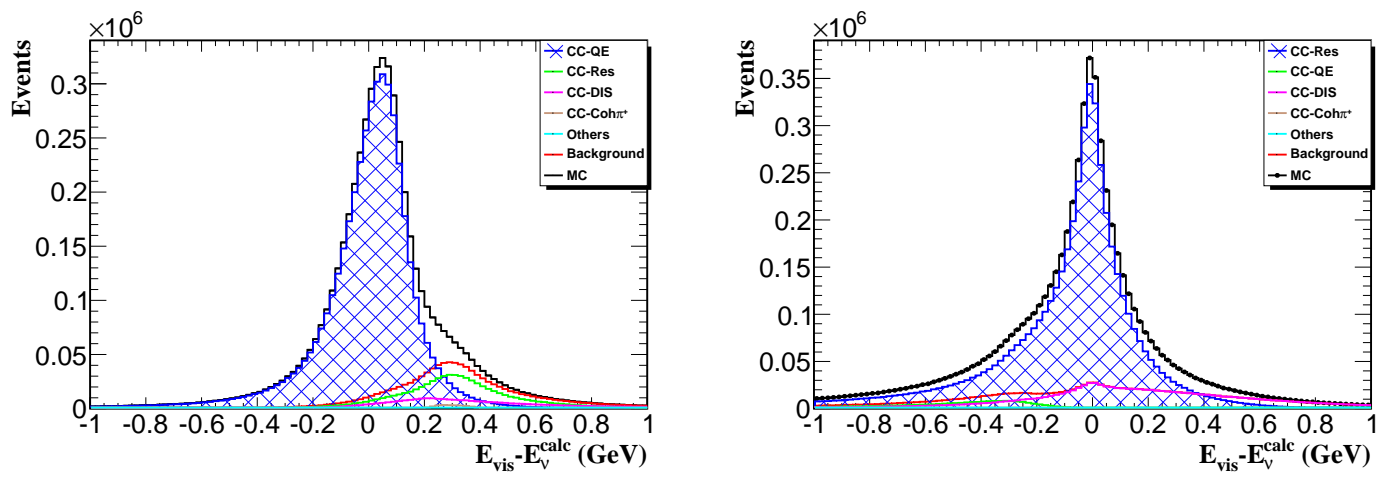

Figure 5: The distribution of $\Delta E$ in CCQE (left) and CCRes (right).

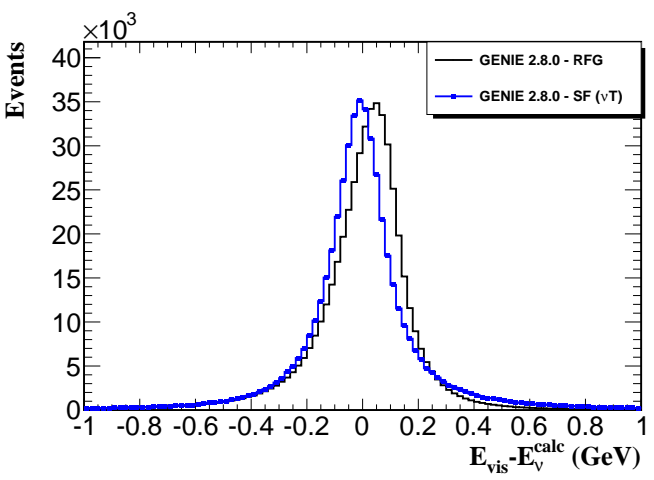

Figure 6: The distribution of $\Delta E$ with different nuclear model. 


\section{Conclusions}

Long-Baseline Neutrino Experiment (Facility), as the third generation neutrino experiment focusing on the determination of neutrino mass hierarchy and $C P$, the control of the systematic uncertainties are the key to achieve its goal. To reach the physics objectives, in particular, sensitivity to discovery of $C P$ violation, at the $5 \sigma$ level, with exposures less than 500 kt.MW.years, a high-capable near detector is a must. Some preliminary studies performed show that Fine-Grained Tracker has the precision and redundancy to constrain the systematic uncertainties below the required level in beam flux, neutrino interaction modeling and detector effects. In addition, the highresolution near detector will also independently conduct precision measurements of quantities such as neutrino-nucleon and neutrino-nuclear cross sections, electroweak parameters, sum-rules and sensitive searches for new physics, like light dark matter, with previously unachievable precision, potentially leading to additional discoveries in neutrino physics.

\section{References}

[1] C. Adams, et al., "The Long-Baseline Neutrino Experiment - Exploring Fundamental Symmetries of the Universe", arXiv:hep-ex/1307.7335.

[2] C. Andreopoulos, et al., Nucl. Instrum. Meth. A614, 87-104 (2010), arXiv:hep-ph/0905.2517.

[3] K. A. Olive, et al., Chin. Phys. C 38, 090001 (2014).

[4] W. J. Marciano and Z. Parsa, J. Phys. G, 29, 2629-2645 (2003), arXiv:hep-ph/0403168.

[5] J. Park, (Ph.D. Thesis), "Direct Measurement of the NuMI Flux with Neutrino-Electron Scattering in MINERVA”, http://lss.fnal.gov/archive/thesis/2000/fermilab-thesis-2013-36.pdf.

[6] S. Mishra, et al., Phys. Rev. Lett. 63, 132-135 (1989).

[7] S. Mishra, et al., Phys. Lett. B252, 170-176 (1990).

[8] P. Vilian, et al., Phys. Lett. B364, 121-126 (1995).

[9] A. A. Aguilar-Arevalo, et al. (MiniBOONE Collaboration), Phys. Rev. D, 81, 092005 (2010).

[10] V. Lyubushkin, et al. (NOMAD Collaboration), Eur. Phys. J. C63, 355 (2009).

[11] C. -M. Jen, A. M. Ankowski, O. Benhar, A. P. Furmanski, L. N. Kalousis, and C. Mariani, Phys. Rev. D 90, 093004 (2004), arXiv:hep-ex/1402.6651.

[12] W. Lerche, et al., Phys. Lett. B78, 510-514 (1978).

[13] V. V. Ammosov, et al. (SKAT Collaboration), Sov. J. Nucl. Phys. 50, 57 (1988).

[14] H. J. Grabosch, et al. (SKAT Collaboration), Z. Phys. C41, 527 (1989).

[15] D. Y. Hongyue, X. C. Tian, and S. R. Mishra, "Resonance Interaction Measurement in NOMAD", NUFACT 2014, University of Glasgow, 25-30, August, 2014. 